\title{
Whole Exome Sequencing: Novel Genetic Polymorphisms in Saudi Arabian Attention Deficit Hyperactivity Disorder (ADHD) Children
}

\author{
Neda Mostafa Bogari ${ }^{1}$, Amr Ahmed Amin ${ }^{2,3}$, Ashwag Aljohani ${ }^{1}$, Ghida Dairi ${ }^{4}$, \\ Mahmoud Zaki El-Readi' ${ }^{2,5}$, Anas Dannoun ${ }^{1}$, Udaya Raja ${ }^{6}$, Mohammad Adil ${ }^{6}$, Nermeen Qutub ${ }^{7,8}$, \\ Suhair Alhelfawi ${ }^{7,9}$, Amal Alobaidi ${ }^{8}$, Derar Alqudah ${ }^{7}$, Hussain Banni ${ }^{1}$, Safaa Yehia Eid ${ }^{2}$, \\ Huda Balto ${ }^{10}$, Faisal Al-Allaf ${ }^{1}$, Mohiuddin Taher ${ }^{1,11}$, Hiba Saed Al-Amoodi'2, \\ Ahmed Fawzy 12 \\ ${ }^{1}$ Department of Medical Genetics, Faculty of Medicine, Umm Al-Qura University, Makkah, KSA; ${ }^{2}$ Biochemistry \\ Department, Faculty of Medicine, Umm Al-Qura University, Makkah, KSA; ${ }^{3}$ Faculty of Medicine, Ain Shams \\ University, Cairo, Egypt; ${ }^{4} \mathrm{Umm}$ Al-Qura University, Makkah, KSA; ${ }^{5}$ Biochemistry Department, Faculty of \\ Pharmacy, Al-Azhar University, Assuit, Egypt; ${ }^{6}$ Integrated Gulf Biosystems, Jeddah, KSA; ${ }^{7}$ Special Needs \\ Department, School of Education, Umm Al-Qura University, Mecca, KSA; ${ }^{8}$ Sinad City for Special Needs, Makkah, \\ KSA; ${ }^{9}$ Institute of Education, University of Reading, Reading, UK; ${ }^{10} \mathrm{Faculty}$ of Medicine, King Abdulaziz \\ University, Jeddah, KSA; ${ }^{11}$ Science and Technology Unit, Umm Al-Qura University, Makkah, KSA; ${ }^{12}$ Division of \\ Human Genetics \& Genome Researches, Department of Molecular Genetics and Enzymology, National Research \\ Centre, Dokki, Egypt
}

Correspondence to: Neda Mostafa Bogari,nmbogari@uqu.edu.sa; Amr Ahmed Amin, aaamin@uqu.edu.sa Keywords: Next Generation DNA Sequencing, Single Nucleotide Polymorphisms, Saudi Children, Attention-Deficit Hyperactivity Disorder, ADHD

Received: January 28, 2019 Accepted: February 25, 2019

Published: February 28, 2019

Copyright $\odot 2019$ by authors and Scientific Research Publishing Inc.

This work is licensed under the Creative Commons Attribution International License (CC BY 4.0).

http://creativecommons.org/licenses/by/4.0/

\section{(c) (1) Open Access}

\section{ABSTRACT}

Background: Attention-deficit hyperactivity disorder (ADHD) is a widespread and debilitating disorder with relatively high prevalence in Saudi Arabia. Neuropsychological and radiological investigations have revealed that there are some differences in the components of the brain regions in children with and without ADHD. In this study we have performed whole exome sequencing (WES) in four non-familial cases of ADHD from Makkah Region to identify the genetic polymorphisms associated with the disease in our Saudi population. Methods: Exome sequencing was carried out using Ion Proton with AmpliSeq Exome library methods, and the data were analysed by Ion Reporter 5.6 software. Results: A total of 33 variants were identified from 222 genes selected from the GWAS catalogue for ADHD associated genes. However, the SNPs we identified in these genes were not reported to be associated with ADHD in previous studies. We have identified 2 novel missense variants; one in c.3451G > T; p. (Ala1151Ser) in ITGA1 gene and another is c.988G > A; p. (Ala330Thr) in 
SPATA13 genes. The variants rs928661, rs11150370 and rs386792899 were the only three variants that appeared on all the 4 patients studied. Six missense variants, rs16841277, rs2228209, rs2230283, rs3741883, rs1716 and rs2272606, were found in 3 different patients, respectively. However, the three documented variants are rs13166360 with bipolar disorder, rs920829 with neuropathic pain, and rs6558702 with schizophrenia. Conclusion: We have identified 2 novel variants in ADHD children. SIFT score of all variants indicates that these substitutions have damaging effects on the protein function. Further screening studies are recommended for confirmation.

\section{INTRODUCTION}

It is commonly accepted that genetic risks for psychiatric disorders, such as attention-deficit hyperactivity disorder (ADHD), schizophrenia, obsessive-compulsive disorder (OCD), bipolar disorder and autism, are conferred by mutations in multiple genes. Attention-deficit hyperactivity disorder (ADHD) is one of the major public health related problems and is a neuro-developmental disorder of childhood [1]. Worldwide about $5 \%$ of children have ADHD [2], and in the Kingdom of Saudi Arabia (KSA) the reported cases of ADHD are 16.4\% [3]. According to Saudi ADHD society (https://adhd.org.sa/en/), an estimated 1.6 million children suffer from ADHD in the kingdom. ADHD is defined by inappropriate levels of inattention, hyperactivity, and impulsivity and it affects boys more than girls [4]. ADHD is associated with hypo-activation of executive functions, including neurocognitive processes, such as inhibition, has widespread learning and memory problems, task switching or set-shifting, and the inability to plan, and pays prolonged attention [5]. The aetiology of ADHD is not clear, having multifactorial aspects [6]; however, recent studies have revealed genetic factors are strongly implicated in the aetiology of ADHD [7]. However, the exact cause of ADHD is not clear; a number of factors can contribute to the development of $\mathrm{ADHD}$, such as genetics, smoking, alcohol abuse, or drug use during pregnancy; exposure to environmental toxins during pregnancy, exposure to environmental toxins during growth and development, low birth weight, and brain injuries are associated with ADHD development. A recent study has indicated that there is a modest association between higher frequency of digital media use and the development of ADHD symptoms; however, further research is required to determine whether this association is causal [8]. Executive function disorder is implicated inmost symptoms of ADHD and is involved in the frontal-subcortical circuits of the brain [9]. On the other hand, the biochemical changes in dopaminergic, adrenergic, and serotonergic systems are associated with the appearance of the symptoms of ADHD [10]. Therefore, the changes in the brain area and neurotransmitter levels contribute to the explanation of the neural dysfunction of ADHD [11]. ADHD is considered one of the familial disorders [12].

Numerous candidate genes for ADHD were implicated by genome-wide association studies. Several studies suggest the involvement of the dopamine neurotransmitter system in the aetiology of ADHD. Specifically, Ribasés et al. (2012) have shown the association between DRD1 and childhood ADHD [13]. According to previous genetic studies, many genes rather than a single gene were found to play a role in the development of ADHD. Polymorphisms in genes, such as VNTR, DAT1, DRD2, DRD3, DRD4, DRD5, DBH, 5-HTT, HTR1B and SNAP2, were reported to be associated with ADHD [14]. Furthermore, genome-wide association studies recently identified common and rare variants and implicated about 391 SNPs with this disease (https://www.ebi.ac.uk/gwas/). Exome analysis has been used to successfully identify diseases causing protein variants in majority of cancers and other diseases; also recently researchers are identifying potential causative mutations associated with major psychiatric diseases such as schizophrenia (SCZ), bipolar disorder, obsessive-compulsive disorder (OCD), major depression disorder (MDD), anxiety disorders (AD), autism, and ADHD. Hawi et al. (2018) have shown that novel DNA variants in the brain derived neurotrophic factor (BDNF) gene increase risks for ADHD using next generation sequencing (NGS) conducted on an Illumina HiSeq2000 platform [15]. Another recent study also has shown that rare and more frequent variants in multiple genes act together in contributing to ADHD risk by combin- 
ing linkage analysis and whole exome sequencing (WES) [16].

ADHD is highly heritable in children with an estimated mean heritability of $76 \%$ [17]. Several studies have suggested that the risk for ADHD may be higher (2 - 8 times) among the first-degree relatives [18]. Consanguineous marriages are common in many Middle Eastern countries, including Saudi Arabia, and around $57.7 \%$ prevalence of consanguinity with first-cousin marriages is the most frequent [19]. This may be one of the reasons for the high incidence of genetic disorders including ADHD, too. The new finding of this study is the screening of Saudi Arabian school children (age > 14 years) in the Makkah (Mecca) region for molecular identification of polymorphisms associated with ADHD response. This will provide a genetic screening map for the molecular genetic determinants of the disease in Saudi children, with the goal of reducing the impact of chronic diseases on health and the economy this kingdom.

\section{METHODS}

\subsection{Study Design and Clinical Presentation}

This study is a retrospective case-study approved by the Medical Ethical Committee of the Faculty of Medicine, Umm Al-Qura University (Ethical approval No HAPO-02-K-012-2017-01-204) and conducted in accordance with the principles of the Declaration of Helsinki. Consent to participation in this study was obtained from the legal guardians of all patients. Four Saudi Arabian paediatric patients with ADHD were recruited in this study; the cases were selected from those scoring a significant degree of ADHD according to the Electronic Observation Questionnaire. The total number of these cases was 106, with variations in several parameters including age (6 - 14 years old), and gender (58 males 54.7\% and 48 females $45.3 \%$ ). Inclusion criteria of being at ages 6 -14, and having met the criteria of primary diagnosis of ADHD based on Diagnostic and Statistical Manual of Mental Disorders (DSM-V) were performed. Any patient of abnormal body mass according to age and gender, and/or having any psychotic or other neurological disorders, and/or having any medical condition conflicting the assessment (e.g. hearing or cardiac abnormal-

ities) were considered as an exclusion criteria.

They were either diagnosed in the clinic by a specialist in the research team, or were previously diagnosed by a specialist. All the cases have met the criteria of Attention-Deficit Hyperactivity Disorder Clinical List (see attached supplementary Table 1) that was derived from the Diagnostic and Statistical Manual of Mental Disorders-fifth edition (DSM-5) [20].

An electronic questionnaire that contained 22 questions was created, it included questions about the previous mental and medical history of the subjects also [21]. Then the questionnaire was distributed to health facilities and centres of developmental disorders in general education. One hundred and six contributors have filled the questionnaire. The parents of the 30 cases out of the 106, matched the required criteria from the electronic questionnaire and were seen by the consultant and answered questions about their child to confirm the diagnosis and determine the severity of the case. Then, the patient was observed to better determine the ADHD symptoms. The confirmation of the diagnosis was done according to the criteria of the Clinical Symptoms of ADHD list [22] where, 10 patients were classified as mild, 15 patients were moderate, and 5 were severe. Each case took about 45 minutes to confirm, and was categorized into one of three groups: ADHD cases, ADHD associated with Autism cases, and ADHD associated with developmental delay cases. Among the 30 cases that were seen in the clinic, 18 of the cases met the criteria of just ADHD excluding any other form of associated diseases.

Table 1. Internal consistency validity for a list of clinical symptoms of attention-deficit hyperactivity disorder.

\begin{tabular}{ccc}
\hline Symptoms & No. of items & Correlation coefficients \\
\hline Attention-deficit & 9 & 0.83 \\
Hyperactivity and impulsivity & 9 & 0.85 \\
\hline
\end{tabular}


The protocol was designed for the attention-deficit and hyperactivity disorder. It consists of 18 parameters divided into two parameters: one for attention-deficit which included 9 phrases, and another is for hyperactivity and impulsivity which included 9 phrases. All the parameters were derived from the Diagnostic and Statistical Manual of Mental Disorders-fifth edition (DSM-5). The list was designed as a 3 -point scale as follows (parameter does not exist $=0$, parameter is partly present $=1$, parameter is fully present $=2$ ). The highest score that may be obtained is 36 , and the lowest score is 0 . A high score of the protocol reflects the presence of the parameter in two places or more, which hinders the performance of the general situation. Our protocol showed high psychometric characteristics, based on the participants' responses to a question on a 3-point frequency scale, values of the Pearson correlation coefficient as a measure of internal consistency validity were calculated between the participants' scores on each dimension and the total score of the scale, as will shown in Table 1.

\subsection{DNA Isolation and Whole Exome Sequencing}

Genomic DNA was extracted from EDTA anticoagulated peripheral venous blood samples using PureLink ${ }^{\mathrm{TM}}$ Genomic DNA Mini Kit by Applied Bio-systems (Catalog number: K182001). Genomic DNA was quantified using Qubit-dsDNA HS (High Sensitivity) Assay Kit (Thermo Scientific). Whole exome sequencing was performed for patient DNA samples using Ion Torrent technology from the Thermo Scientific. 50 - $100 \mathrm{ng}$ of the genomic DNA was subjected to enriched library preparation with an Ion AmpliSeq $^{\mathrm{TM}}$ Library Kit Plus kit (Cat No. 4488990) with mean amplicon size of 215 bpand ion AmpliSeqTM Exome RDY Panel 1x8 containing primer pools (Cat. No. 4489838, Life Technologies, USA) kit following the manufacturer's protocol, and then quantified using qPCR using Ion Library TaqMan quantitation kit (Cat. No. 4468802). Each sample was assigned a distinct barcode using the Ion-Xpress barcode kit (Cat. No. 4471250). Barcoded libraries were diluted to $100 \mathrm{pmol}$, pooled samples libraries and subjected to template preparation on Ion sphere particles (ISP) using the Ion One Touch 2 System and Ion PI HI-Q Template OT2 200 kit (Cat. No. A26434). Each templated ISP was loaded on the Ion PI v3 chip (Cat. No. A26770) and sequenced on the Ion Proton instrument (Life Technologies, USA) using the Ion PI Hi-Q Sequencing Kit (Cat. No. A26772). The alignments were performed by a Torrent Suite v4.2. Filtering low quality reads, adapter removing followed alignment against the human reference genome (hg19 build); bio-informatic analysis was performed using Torrent Suite Software (v5.10.1.0), and the variants were called using the Torrent Variant Caller plugin (v5.10), and then imported into the Ion Reporter software (v5.10) for the annotation. Gene variants located within exons, introns, predictive splice sites and UTRs were identified with less than $1 \%$ minor allele frequency (MAF). Functional exons variants were also identified as frame-shift, synonymous, missense, nonsense and stop loss.

\subsection{Biostatistical Analysis and Bio-Informatics}

The total number of genes is 271 which were selected form the ADHD the genome-wide association study (GWAS) data base. However, only 222 genes were used in filtration in this study because 49 of the genes which did not match the ion torrent library kit (attached supplementary Table 2). ADHD included exonic variants nonsense, missense, frame-shift insertion, frame-shift deletion, and using the Ion Reporter v5.6 annotation tool for functional consequences for genes and variants.

Table 2. Summary of ADHD patients' characteristics.

\begin{tabular}{cccccc}
\hline Patient number & Age at diagnosis & Sex & Attention deficit score & Hyperactivity & Total score \\
\hline 1 (case) & 11 & F & 9 & 9 & 18 \\
2 (case) & 6 & M & 7.5 & 9 & 16.5 \\
3 (case) & 9 & F & 9 & 7.5 & 16.5 \\
4 (case) & 11 & M & 7 & 8 & 15 \\
\hline
\end{tabular}




\section{RESULTS}

Table 1 shows values of the Pearson correlation coefficient between the Attention-Deficit dimension, hyperactivity and impulsivity dimension and the total scores, which are 0.83 and 0.85 , respectively. The values are statistically significant at $0.01 \mathrm{p}$-value.

To examine the reliability of the clinical symptoms of the ADHD list, Cronbach's coefficient between each dimension of the list and the list total score were calculated. Cronbach's value for the instrument is 0.85 while it is 0.80 for the Attention-Deficit dimension and 0.82 for the hyperactivity and impulsivity dimension, which are statistically significant.

Selection of samples for whole exome analysis: 4 patient samples (the most severe cases) from our previously mentioned 18 cases of just ADHD were selected for exome sequencing for this study. The 4 patients' selection was based on diagnosis, severity, and age. The criteria and patient phenotypic characteristics are summarized in Table 2.

In this study, DNA samples from four children with ADHD were sequenced for whole exome analysis (Sample 1 - 4; Table 2) samples were diagnosed for ADHD according to the Diagnostic and Statistical Manual of Mental Disorders-fifth edition (DSM-5 Diagnostic Criteria). Half of cases were male (50\%) in an age range of 6 - 11 years. The average read depth for the targeted base coverage was $82 \times$, with $94 \%$ of targeted regions covered at greater than $20 \times$.

Almost, more than 160,000 variants per VCF files were annotated from all the samples. In this study a total of 51,920,56,215, 55,747 and 54,664 variants were found in the children with ADHD. In Table 3 we have shown the filtered variants applying the filters to retrieve pathogenic variants, the filters including exonicvariants, sorting intolerant from tolerant (SIFT) score $<0.05$ and PolyPhen score of $>0.5$.

Thirty-three variants were identified following filtering for potential functionality, 31 of which had been identified previously in the dbSNP and 2 were novel. The 2 novelmissense variants identified in the study were found to be of ITGA1 (chr5:52243247) c.3451G > T; p. (Ala1151Ser) in one patient, and in SPATA13 (spermatogenesis-associated protein 13) at chr13:24798055 locus c. 988G > A; p. (Ala330Thr) in another patient. Six missense variants (rs16841277, rs2228209, rs2230283, rs3741883, rs1716, rs2272606) were found in 3 patients respectively. Other seven missense variants (rs6558702, rs920829, rs149000560, rs2279574, rs11109968, rs9935113, rs7191351) were found in only two of the four patients. Eighteen other variants were identified in only one patient each (Table 3). The quality statistics of the variants is shown in Table 4. All variants show very low SIFT score indicating these substitutions in the variants have damaging effect on the protein function. Many variants have high PolyPhen score, suggesting variants with score in this range are more confidently predicted to be damaging.

SIFT score predicts protein function deleterious effects based on amino acid substitution. Scores ranges from $0.0-1.0$, variants with scores less than 0.05 are considered as deleterious, a SIFT value of 1.0 is considered as benign. The heat map generated 56 variants from 27 genes (Figure 1).

Clustering Algorithms to visually identify patterns based upon the scoring of variant impact, the rows and columns of a heat map are often sorted by hierarchical clustering trees. Heat maps of different SNPs analysed using SIFT score and hierarchical clustering of genes in 4 ADHD patients is shown in Figure 1.

\section{DISCUSSION}

In our study we found 33 variants in 222 commonly associated genes with ADHD listed in GWAS database. Out of these 31 SNPs had been reported previously in the dbSNP and 2 SNPs were novel. All the target genes we have selected from the GWAS data base for the analysis in this study (supplementary Table 2) are known to be linked to ADHD (http://adhd.psych.ac.cn); however, we have detected many additional SNPs which are new, because previously these SNPs were not linked to ADHD. This indicates our finding is a new to demonstrate the association of known SNPs with the ADHD in Saudi children. There was no association was demonstrated with the ADHD and our newly detected SNPs in the previous study, information about 14 previously reported variants (rs13166360, rs150179494, rs6558702, rs920829, rs149000560, rs2279574, rs2230283, rs3741883, rs928661, rs2306022, rs45551636, rs9935113, rs1716, and 
Table 3. Rare and novel variants identified by exome sequencing in Saudi Arabian ADHD patients.

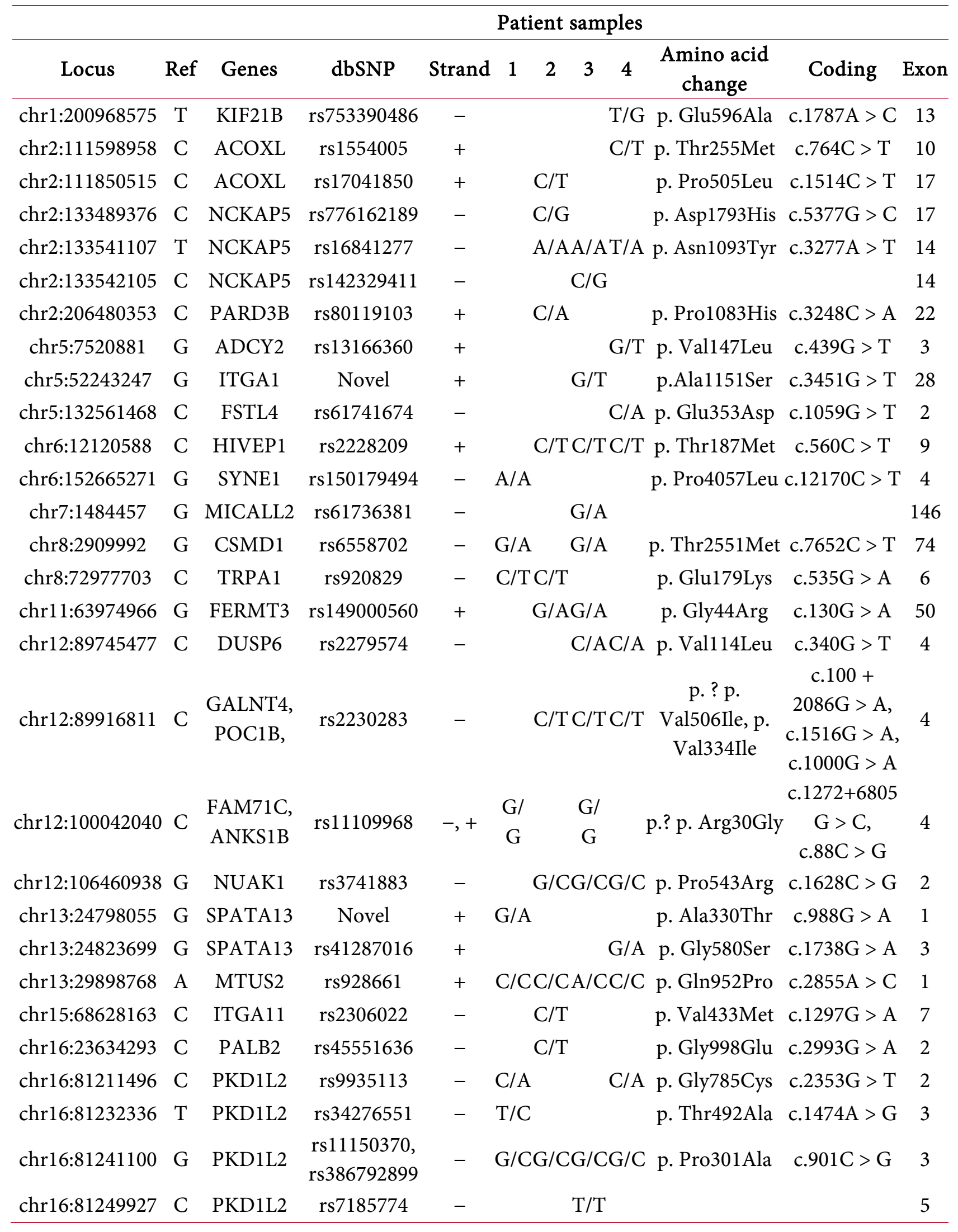




\section{Continued}

\begin{tabular}{cccccccccc}
\hline chr16:81249954 & T & PKD1L2 & rs7191351 & - & T/A & A/A & p. Gln120Leu & c.359A $>$ T & 12 \\
chr16:82660738 & C & CDH13 & rs753448816 & + & C/A & p. Pro26Thr & c.76C $>$ A & 9 \\
chr17:3632836 & G & ITGAE & rs1716 & - & G/A & G/AG/A & p. Arg950Trp & c. $2848 C>T$ & 4 \\
chr17:3657159 & C & ITGAE & rs2272606 & - & T/T T/T & C/T & p. Arg482Gln & c.1445G $>$ A & 14 \\
\hline
\end{tabular}

Table 4. Quality Statistics of Rare and novel variants identified by exome sequencing in ADHD patients.

\begin{tabular}{|c|c|c|c|c|c|c|c|c|c|c|c|}
\hline \multirow[b]{2}{*}{ Genes } & \multirow[b]{2}{*}{$\mathrm{dbSNP}$} & \multirow[b]{2}{*}{$\begin{array}{l}\text { Variant } \\
\text { frequency } \\
\text { in our } \\
\text { study }\end{array}$} & \multirow[b]{2}{*}{$\frac{a}{\stackrel{0}{a}}$} & \multirow[b]{2}{*}{ 岀 } & \multirow[b]{2}{*}{ 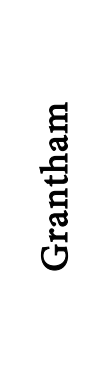 } & \multirow[b]{2}{*}{ 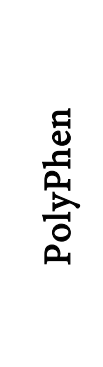 } & \multirow[b]{2}{*}{ 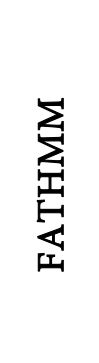 } & \multicolumn{4}{|c|}{ Comparison of Var Freq [62] } \\
\hline & & & & & & & & 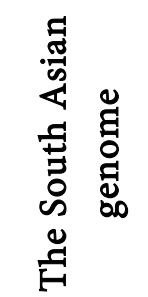 & Z & 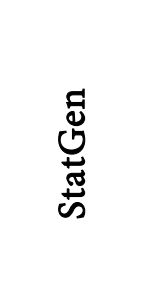 & 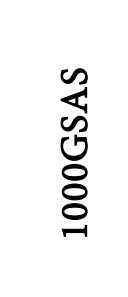 \\
\hline KIF21B & rs753390486 & 0.25 & 6.97 & 0.03 & 107 & 0.993 & & & & & \\
\hline ACOXL & rs 1554005 & 0.25 & 0.15 & 0 & 81 & 0.672 & 0.466 & 0.24303 & 0.20789 & 0.16667 & 0.22279 \\
\hline ACOXL & rs17041850 & 0.25 & 2.04 & 0.03 & 98 & 0.978 & 0.392 & 0.00952 & 0.10932 & 0.01389 & 0.02156 \\
\hline NCKAP5 & rs776162189 & 0.25 & 6.14 & 0 & 81 & 1 & & & & & \\
\hline NCKAP5 & rs16841277 & 0.75 & 0.5 & 0 & 143 & 0.969 & 0.259 & 0.25542 & 0.22222 & 0.16667 & 0.22793 \\
\hline NCKAP5 & rs142329411 & 0.25 & -0.69 & 0.02 & 58 & 0.589 & & & & & 0.0 \\
\hline PARD3B & rs80119103 & 0.25 & 1.09 & 0 & 77 & 0.606 & & & 0.01254 & & 0.000 \\
\hline ADCY2 & rs 13166360 & 0.25 & 6 & 0 & 32 & 0.598 & & 0.15635 & 0.08065 & 0.04167 & 0.10780 \\
\hline ITGA1 & Novel & 0.25 & 3.52 & 0.03 & 99 & 0.998 & & & & & \\
\hline FSTL4 & rs61741674 & 0.25 & 0.58 & 0 & 45 & 0.999 & & 0.02632 & 0.01434 & & 0.01027 \\
\hline HIVEP1 & rs2228209 & 0.75 & 0.64 & 0 & 81 & 0.982 & & 0.26471 & 0.23477 & 0.30556 & 0.28234 \\
\hline SYNE1 & rs150179494 & 0.25 & 6.51 & 0.01 & 98 & 0.936 & 0.852 & & 0.00358 & & 0.000 \\
\hline MICALL2 & rs61736381 & 0.25 & 2.79 & 0 & 101 & 1 & & & & & 0.0 \\
\hline CSMD1 & rs6558702 & 0.5 & 0.96 & 0.04 & 81 & 0.653 & & 0.75160 & 0.70072 & 0.81944 & 0.70739 \\
\hline TRPA 1 & rs920829 & 0.5 & 6.72 & 0.05 & 56 & 0.994 & 0.984 & 0.17619 & & 0.25000 & 0.14374 \\
\hline FERMT3 & rs149000560 & 0.5 & 8.53 & 0 & 125 & 1 & & 0.00595 & 0.00717 & & 0.00000 \\
\hline DUSP6 & rs2279574 & 0.5 & 5.81 & 0.03 & 32 & 0.78 & 0.977 & 0.47030 & 0.49104 & 0.52778 & 0.48665 \\
\hline $\begin{array}{c}\text { GALNT4, } \\
\text { POC1B, }\end{array}$ & rs2230283 & 0.75 & 7.43 & 0.02 & $\begin{array}{l}29.0 \\
29.0\end{array}$ & 0.896 & 0.988 & 0.18731 & 0.25627 & 0.23611 & 0.16632 \\
\hline $\begin{array}{l}\text { FAM71C, } \\
\text { ANKS1B }\end{array}$ & rs11109968 & 0.5 & -0.01 & 0 & 125 & 0.971 & & 0.20588 & 0.21147 & 0.16667 & 0.17556 \\
\hline NUAK1 & rs3741883 & 0.75 & 3.53 & 0 & 103 & 0.93 & 0.945 & 0.26471 & 0.25986 & 0.19444 & 0.27002 \\
\hline SPATA13 & Novel & 0.25 & 3.65 & 0.01 & 58 & 0.855 & & & & & \\
\hline SPATA13 & rs41287016 & 0.25 & 1.64 & 0 & 56 & 0.817 & & 0.01746 & 0.02688 & 0.02778 & 0.01232 \\
\hline MTUS2 & rs928661 & 1 & 1.89 & 0 & 76 & 1 & 0.198 & 0.87926 & 0.81720 & 0.87500 & 0.88090 \\
\hline
\end{tabular}




\section{Continued}

\begin{tabular}{|c|c|c|c|c|c|c|c|c|c|c|}
\hline ITGA11 & rs2306022 & 0.25 & -0.18 & 0.03 & 21 & 0.928 & 0.1 & 0.072330 .04659 & 0.05556 & 0.06057 \\
\hline PALB2 & rs 45551636 & 0.25 & 2.85 & 0 & 98 & 1 & 0.911 & 0.01111 & $\begin{array}{l}0.01254 \\
0.00205\end{array}$ & \\
\hline PKD1L2 & rs9935113 & 0.5 & 3.89 & 0 & 159 & 1 & 0.769 & 0.269350 .17742 & 0.19444 & 0.28131 \\
\hline PKD1L2 & rs34276551 & 0.25 & 4.29 & 0.01 & 58 & 0.775 & & 0.004760 .00179 & & 0.00308 \\
\hline PKD1L2 & $\begin{array}{l}\text { rs11150370, } \\
\text { rs386792899 }\end{array}$ & 1 & 6.05 & 0.01 & 27 & 0.951 & 0.967 & 0.491480 .57885 & & 0.50411 \\
\hline PKD1L2 & rs7185774 & 0.25 & 3.98 & 0.01 & 94 & 0.998 & & 0.195510 .13262 & 0.15278 & 0.14682 \\
\hline PKD1L2 & rs7191351 & 0.5 & 1.17 & 0 & 113 & 0.763 & & 0.436340 .46416 & 0.43056 & 0.40862 \\
\hline $\mathrm{CDH} 13$ & rs753448816 & 0.25 & 3.5 & 0 & 38 & 1 & & & & \\
\hline ITGAE & rs1716 & 0.75 & -0.21 & 0.02 & 101 & 0.999 & 0.022 & $0.52063 \quad 0.28674$ & 0.55556 & 0.53901 \\
\hline ITGAE & rs 2272606 & 0.75 & -0.22 & 0 & 43 & 0.783 & 0.023 & 0.407940 .47491 & 0.3333 & 0.41170 \\
\hline
\end{tabular}

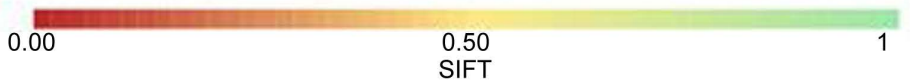

Figure 1: Detail of Sift score for all the 4 ADHD cases

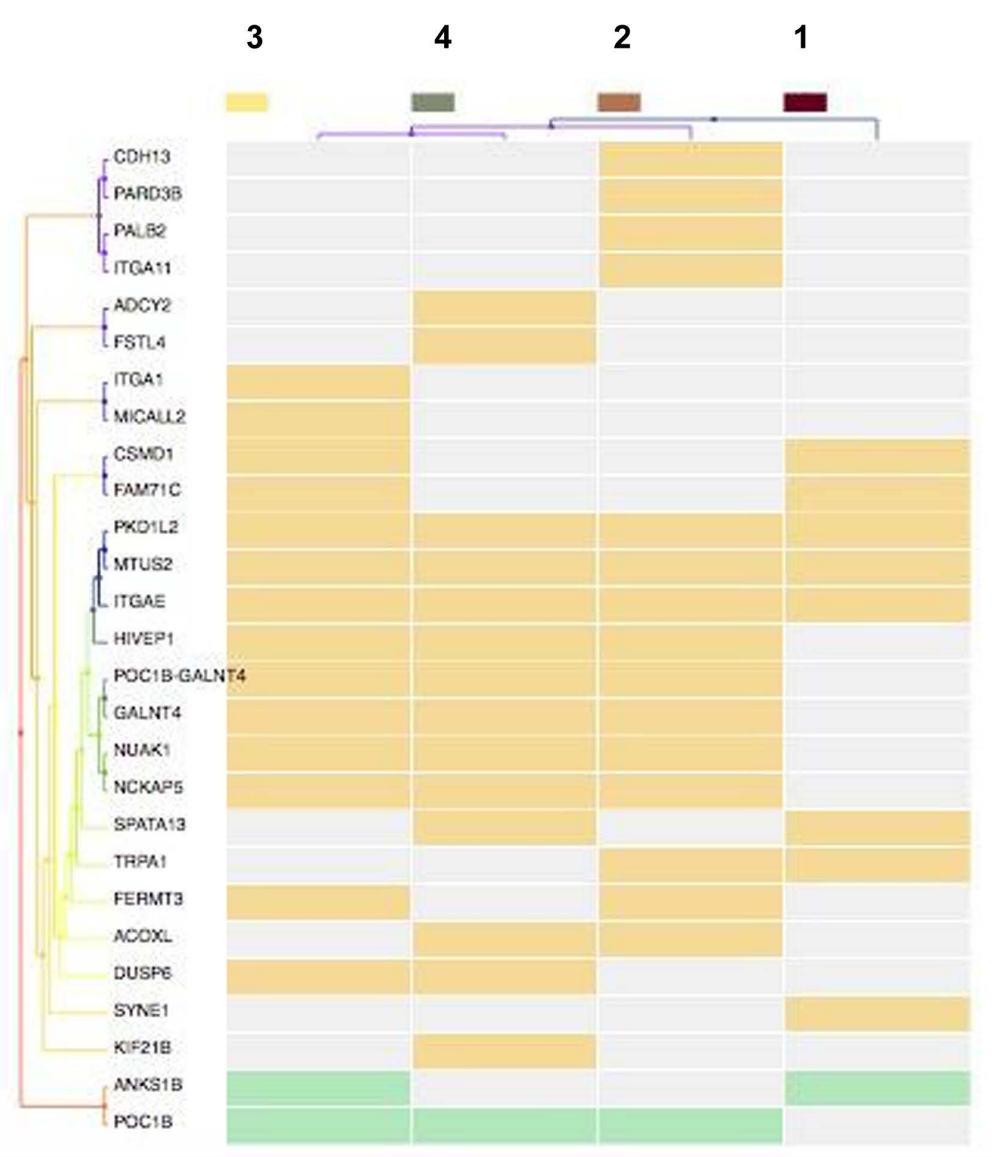

Figure 1. The details of SIFT scores. 
rs2272606). The 2 novel missense variants identified in the study were found to be encoded for ITGA1 (chr5:52243247) c.3451G > T; p. Ala1151Ser in one patient, and in SPATA13 (chr13:24798055) gene c. 988G > A; p. Ala330Thr in one patient, this gene also known as ASEF2 and serves as a GEF for both CDC42 and RAC1 in cell culture and that by binding to APC (adenomatosis polyposis coli) increases its GEF activity, and modulates the cell migration [23]. Also, we have found a missense5'-UTR variant G $>$ A (rs41287016) in SPATA13 gene which has been reported previously. One SNP in ADCY2 (Adenylate cyclase-2) rs13166360 is associated with bipolar disorder [24]. Another SNP we found in the gene DUSP6 (rs2279574) also known to be associated with bipolar disorder [25].

The identified polymorphisms in this study have high variant frequencies $(0.25-1)$. In addition, all amino acid substitutions for the mutant nucleotide are predicted deleterious according to the SIFT scores $(\geq 0.05)$. One variant in each gene, viz., MTUS2 (rs928661) c.2855A > C; p. Gln952Pro; and another variant in PKD1L2 (rs11150370) c.901C > G; p. Pro301Ala; were found in all the 4 cases respectively. Six missense variants (rs16841277, rs2228209, rs2230283, rs3741883, rs1716, rs2272606) were found in 3 patients. Seven missense variants (rs6558702, rs920829, rs149000560, rs2279574, rs11109968, rs9935113, rs7191351) were found in two of the four patients.

Four different SNPs were identified in our study for the NCK Associated Protein 5 (NCKAP5) gene on ch:2; the diseases associated with NCKAP5 include neurological disorder, hypersomnia which affiliates many tissues including brain, causing behaviour and neurological disorders [26]. The National Sleep Foundation states that ADHD is linked with a variety of sleep problems. For example, children with ADHD had increased rates of daytime sleepiness, had shorter sleep durations, more sleep problems on weekdays, anxiety or depression aggravated these sleep complications in the ADHD children [27]. Another study found that children with ADHD had signs of sleep-disordered breathing such as habitual snoring, habitual snoring may be three times higher in children with ADHD (Sleep and neurobehavioral characteristics of 5- to 7-year-old children with parentally reported symptoms of ADHD [28].

The ITGAE gene or integrin subunit alpha E belongs to the Integrin alpha subunits (ITGA) family. It functions as a receptor for E-cadherin. This protein mediates adhesion of intra-epithelial T-lymphocytes to epithelial cell monolayers and may be involved in the activation of intestinal intraepithelial lymphocytes (IEL) [29]. It is expressed in multiple tissues including IEL, bone marrow and brain. ITGA11 (integrin subunit alpha 11) is another member of the Integrin alpha subunits (ITGA) family. It is a collagen receptor that is expressed in muscle tissues, and it plays an important role in attaching muscle tissue to the extracellular matrix [30]. Both ITGAE and ITGA11 are involved in cell adhesion. SNP rs220470 in the ITGAE gene and SNP rs7164335 in ITGA11 were present in the 30 top hit single SNPs in a study conducted using independent DNA pools from 343 ADHD-affected adults and 304 controls for association analyses of the ADHD diagnostic phenotype [31].

However, in this study we identified two SNPs in ITGAE (integrin, alpha-E) and one SNP in ITGA11 (integrin, alpha-11) that may have a role in causing ADHD, which are: rs1716, and rs2272606 in ITGAE gene and one SNP rs2306022 in ITGA11.However, these three SNPs are not implicated in ADHD development previously. The SNP rs1716 was previously associated with a gastrointestinal stromal tumour subtype [32] and increased risk of melanoma along with SNP rs2272606 [33], while SNP rs2306022 of ITGA11 was implicated in increasing risk for lung cancer and fibrosis [29]. In three of the patients we have detected a SNP rs3741883 located on the NAUK1 (AMPK-related protein kinase 5; ARK5 or KIAA0537) gene. The NUAK family, SNF1-like kinase gene is highly expressed in heart and brain and involved in several processes that include tumour progression, cell proliferation, cell adhesion, senescence and regulation of cell ploidy [34]. Two SNPs located on NUAK1 (rs6539247 and rs2569973) have been detected in a recent study done in ADHD adult patients, however, in the present study in children with ADHD these two SNPs were not found [35], evidence suggests that the NAUK1 gene was associated with cortical amyloid- $\beta$ protein which plays an essential role in Alzheimer's disease [36], it might also be involved in attention performance in persistent ADHD since Alzheimer's disease is relevant in the context of ADHD.

Additionally, previous studies have identified SNP rs753390486 in KIF21Bgene (kinesin family member 21b; KIF21B or KIAA0449), which was also found in the present study in one ADHD patient. 
This gene is expressed in high level in the CNS, mostly in the dendrites of neurons [37]. The KIF21B gene is a member of the kinesin superfamily that contains 45 genes known as kinesins, divided into 15 different families. Genetic variation in some members of the kinesin family has been linked to neurodegenerative diseases such as Alzheimer's disease, amyotrophic lateral sclerosis (ALS) and Huntington's disease [38]. The KIF21B gene was previously associated with multiple sclerosis [39]. Our results also found five SNPs located on the PKD1L2 gene which are: rs9935113, rs34276551, rs11150370, rs7185774 and rs7191351, this gene belongs to the polycystin family and is expressed in tissues including placenta, mammary gland, liver, lung, skeletal muscle, brain and testis [40]. PKD1L2 may function as an ion-channel regulator and as a G-protein-coupled receptor [41].

Genetic studies have established that several genes have been associated with ADHD such as ACOXL, PADR3B, HIVEP1, TRPA1 and SYNE1. Among these genes, SYNE1 gene contributes to multiple genetic disorders, specifically neuromuscular disorders [42]. The SYNE1 gene is mainly expressed in skeletal muscle cells, therefore, mutations in SYNE1 could alter muscular functions [43]. Mutations in SYNE1 have been linked with numerous cases of autism, bipolar disorder and ARCA1 [42, 44]. It has been identified as one of the top risk genes in causing ADHD according to GWAS studies [45]. Our findings from this study revealed a SNP (rs150179494) in the SYNE1 gene. These results suggest the association between these SNPs and ADHD occurrence. Also, there are no current studies on the effects of the other SNPs in the SYNE1 gene on ADHD development. Furthermore, several studies have linked the ACOXL gene to certain types of tumours. ACOXL showed no levels of gene expression in almost $72 \%$ of prostate tumours [46]. The exact function of the ACOXL gene is largely undefined; however, loci in this gene are thought to participate in causing alopecia areata [35]. Our study has suggested the contribution of four SNPs (rs779762950, rs77331476, rs1554005, rs17041850) in the ACOXL gene that could cause ADHD. Further studies are required to explore the role of the ACOXL gene and the effects of these SNPs in ADHD and other mental disorders.

Another gene, which is thought to be related to ADHD, is the PARD3 (partitioning-defective protein 3 ) gene codes for a protein that is involved in exon splicing and neuronal polarity [47]. Among the PARD gene family, the PARD3B gene, located on the 2q33.33 region [39], has been linked with nicotine dependence defined by the FTND (Fagerström Test for Nicotine Dependence) [48]. Our findings revealed a novel SNP (rs80119103) to be responsible for increasing the susceptibility of ADHD. A recent study reported an association between PARD3 and schizophrenia and ADHD [49], and it has been observed in adults with various forms of disinhibitory psychopathology and antisocial behaviour as well [50]. TRPA1 is a member of the transit receptor potential family (TRPA1), and it was suggested to have a role in several sensing reactions. A study that was performed on knockout mice has shown a relationship between TRPA1 and the transduction of chemical and mechanical stimuli through nociceptor sensory neurons [51]. According to a study that has investigated the role of TRPA1, it is suggested that this gene has an essential role in inflammation and neuropathic pain [52]. Our study identified one of the SNPs in TRPA1 (rs920829) as a risk factor for ADHD. This SNP has been previously presented in another study as a key player in causing asthma in children [53]. The HIVEP1 gene encodes for one of the transcription factors that belong to the ZAS family. It binds to motifs that regulate HIV gene expression [54]. A study has shown that the HIVEP1 gene was one of the highest ranked-genes to be related to mental disorders such as schizophrenia [37], bipolar disease (BP) and post-traumatic stress disorder (PTSD) [55]. The findings from our study have revealed an SNP (rs2228209) in the HIVEP1 gene that could potentially be linked to ADHD.A body of evidence has shown that SNPs in the integrin alpha 1 ITGA1 gene may cause ADHD. A study conducted on 1033 ADHD patients, (age ranging from 6 to 16 years). Four SNPs have been reported in the ITGA1 gene located on chromosome 5 (rs1979398, rs16880453, rs1531545 and rs4074793 [56]. ITGA1 gene has a role in cell attachment and neurite outgrowth as well as in peripheral nerve regeneration [57] however, the exact mechanism ofITGA1 polymorphisms is still not clearly understood in ADHD. Consistent with this study, our data explored the association of the ITGA1 gene at chromosome 5 in position 5:2243247 with ADHD. It has been reported that in ITGAE a SNP rs220470 (intronic variant) was associated with ADHD in Caucasians [31]. To our knowledge, this finding is novel and these SNPs have not been reported in the 
literature. Two Integrin subunits including alpha and beta have been known to have a role in the development of the neurological system by mediating many neural cell functions [58].

In addition, our study has identified SNP on the Fermitin family homolog 3FERMT3 gene on chromosome 11 (rs149000560), another study done in 465 trioshas identified different SNP on the same gene (rs12575642) [59]. This missense variant (rs149000560) is responsible for the leukocyte adhesion deficiency-1/variant syndrome [60]. Taken together, the data suggested that these SNPs might have an association with ADHD susceptibility. On the other hand, our study has identified three SNPs in the NCK Associated Protein 5; NCKAP5 gene (rs776162189, rs16841277, and rs142329411) which may be related to the ADHD phenotype. Indeed, previous study has demonstrated a set of risk genes or copy number variations (CNVs) involved in the autism spectrum, disorder as in human subjects, one of which was the NCKAP5 gene [61]. Our exome sequencing has shown SNPs in the POC1B-GALNT4 and FAM71CANKS1Bgenes, which may have a role in causing ADHD, these SNPs were rs2230283 and rs11109968, respectively. However, to our knowledge the significant association of these two SNPs with ADHD has not been previously reported.

\section{CONCLUSION}

We have identified two novel missense mutations in the ITGA1 and SPATA13 genes respectively in two different ADHD children with exome sequencing analysis. These two novel mutations, c.988G > A, p. Ala330Thr, in SPATA13, and c.3451G > T, p. Ala1151Ser, in ITGA1, have not been reported so far in literature. Our current results revealed mutations which had not previously been linked with ADHD within the database. However, the three documented variants are rs13166360 with bipolar disorder, rs920829 with neuropathic pain, and rs6558702 with schizophrenia. This study recommends screening these in ADHD cases to confirm the prevalence in the Saudi population. It is recommended for future studies to examine the four variants in detail. The strength of our current study is that we have selected satisfactory diagnosis severity ADHD cases in the same region. However, conducting of further studies with larger number of patients is necessary to confirm the ADHD associated SNPs in Saudi Children.

\section{ACKNOWLEDGEMENTS}

This work was supported financially by the Deanship of Scientific Researchat Umm Al-Qura University (Grant Code: 15-MED-3-1-0062) to Dr. Neda Bogari. We are thankful to Mr. Ehab M. Melibary and Mr. Sami M. Kalantan for their technical in this study, Miss Renad Y. Natto, Miss Dema N. Bogari, and Mr Safar A. Almalki for the continuous support. We all are deeply appreciative for Mr. Abdulmonim Gowda, of Integrated Gulf Biosystems. We would like to acknowledge the Albaseera Center and Successful Communication Center, Khawla Bint Khuwailad Center and Center of Optimism.

\section{SOURCE OF FUND}

This work was supported financially by the Deanship of Scientific Researchat Umm Al-Qura University (Grant Code: 15-MED-3-1-0062) to Dr. Neda Bogari

\section{CONFLICTS OF INTEREST}

We wish to confirm that there are no known conflicts of interest associated with this publication and there has been no significant financial support for this work that could have influenced its outcome.

We confirm that the manuscript has been read and approved by all named authors and that there are no other persons who satisfied the criteria for authorship but are not listed. We further confirm that the order of authors listed in the manuscript has been approved by all of us.

We confirm that we have given due consideration to the protection of intellectual property associated with this work and that there are no impediments to publication, including the timing of publication, with respect to intellectual property. In so doing we confirm that we have followed the regulations of our insti- 
tutions concerning intellectual property.

\section{REFERENCES}

1. Tripp, G. and Wickens, J.R. (2009) Neurobiology of ADHD. Neuropharmacology, 57, 579-589. https://doi.org/10.1016/j.neuropharm.2009.07.026

2. Spencer, T.J., Biederman, J. and Mick, E. (2007) Attention-Deficit/Hyperactivity Disorder: Diagnosis, Lifespan, Comorbidities, and Neurobiology. Journal of Pediatric Psychology, 32, 631-642. https://doi.org/10.1093/jpepsy/jsm005

3. AlZaben, F.N., Sehlo, M.G., Alghamdi, W.A., Tayeb, H.O., Khalifa, D.A., Mira, A.T., Alshuaibi, A.M., Alguthmi, M.A., Derham, A.A. and Koenig, H.G. (2018) Prevalence of Attention Deficit Hyperactivity Disorder and Comorbid Psychiatric and Behavioral Problems among Primary School Students in Western Saudi Arabia. Saudi Medical Journal, 39, 52-58. https://doi.org/10.15537/smj.2018.1.21288

4. Skogli, E.W., Teicher, M.H., Andersen, P.N., Hovik, K.T. and Oie, M. (2013) ADHD in Girls and Boys-Gender Differences in Co-Existing Symptoms and Executive Function Measures. BMC Psychiatry, 13, 298. https://doi.org/10.1186/1471-244X-13-298

5. Willcutt, E.G., Doyle, A.E., Nigg, J.T., Faraone, S.V. and Pennington, B.F. (2005) Validity of the Executive Function Theory of Attention-Deficit/Hyperactivity Disorder: A Meta-Analytic Review. Biological Psychiatry, 57, 1336-1346. https://doi.org/10.1016/j.biopsych.2005.02.006

6. Faraone, S.V., Asherson, P., Banaschewski, T., Biederman, J., Buitelaar, J.K., Ramos-Quiroga, J.A., et al. (2015) Attention-Deficit/Hyperactivity Disorder. Nature Reviews Disease Primers, 1, 15020. https://doi.org/10.1038/nrdp.2015.20

7. Biederman, J., Petty, C.R., Wilens, T.E., Fraire, M.G., Purcell, C.A., Mick, E., Monuteaux, M.C. and Faraone, S.V. (2008) Familial Risk Analyses of Attention Deficit Hyperactivity Disorder and Substance Use Disorders. American Journal of Psychiatry, 165, 107-115. https://doi.org/10.1176/appi.ajp.2007.07030419

8. Ra, C.K., Cho, J., Stone, M.D., De La Cerda, J., Goldenson, N.I., Moroney, E., Tung, I., Lee, S.S. and Leventhal, A.M. (2018) Association of Digital Media Use with Subsequent Symptoms of Attention-Deficit/Hyperactivity Disorder among Adolescents. JAMA, 320, 255-263. https://doi.org/10.1001/jama.2018.8931

9. Cortese, S., Kelly, C., Chabernaud, C., Proal, E., Di Martino, A., Milham, M.P. and Castellanos, F.X. (2012) Toward Systems Neuroscience of ADHD: A Meta-Analysis of 55 fMRI Studies. The American Journal of Psychiatry, 169, 1038-1055. https://doi.org/10.1176/appi.ajp.2012.11101521

10. Kooij, S.J., Bejerot, S., Blackwell, A., Caci, H., Casas-Brugué, M., Carpentier, P.J., Edvinsson, D., Fayyad, J., Foeken, K., Fitzgerald, M., et al. (2010) European Consensus Statement on Diagnosis and Treatment of Adult ADHD: The European Network Adult ADHD. BMC Psychiatry, 10, 67.

https://doi.org/10.1186/1471-244X-10-67

11. Cortese, S. (2012) The Neurobiology and Genetics of Attention-Deficit/Hyperactivity Disorder (ADHD): What Every Clinician Should Know. European Journal of Paediatric Neurology, 16, 422-433. https://doi.org/10.1016/j.ejpn.2012.01.009

12. Mathews, C.A. and Grados, M.A. (2011) Familiality of Tourette Syndrome, Obsessive-Compulsive Disorder, and Attention-Deficit/Hyperactivity Disorder: Heritability Analysis in a Large Sib-Pair Sample. Journal of the American Academy of Child and Adolescent Psychiatry, 50, 46-54. https://doi.org/10.1016/j.jaac.2010.10.004

13. Ribasés, M., Ramos-Quiroga, J.A., Hervás, A., Sánchez-Mora, C., Bosch, R., Bielsa, A., Gastaminza, X., Lesch, K.P., Reif, A., Renner, T.J., et al. (2012) Candidate System Analysis in ADHD: Evaluation of Nine Genes Involved in Dopaminergic Neurotransmission Identifies Association with DRD1. The World Journal of Biological Psychiatry, 13, 281-292. https://doi.org/10.3109/15622975.2011.584905 
14. Ballon, N., Leroy, S., Roy, C., Bourdel, M.C., Olie, J.P., Charles-Nicolas, A., Krebs, M.O. and Poirier, M.F. (2007) Polymorphisms TaqI A of the DRD2, BalI of the DRD3, Exon III Repeat of the DRD4, and 3' UTR VNTR of the DAT: Association with Childhood ADHD in Male African-Caribbean Cocaine Dependents? American Journal of Medical Genetics Part B: Neuropsychiatric Genetics, 144B, 1034-1041. https://doi.org/10.1002/ajmg.b.30540

15. Hawi, Z., Cummins, T.D., Tong, J., Arcos-Burgos, M., Zhao, Q., Matthews, N., Newman, D.P., Johnson, B., Vance, A., Heussler, H.S., et al. (2017) Rare DNA Variants in the Brain-Derived Neurotrophic Factor Gene Increase Risk for Attention-Deficit Hyperactivity Disorder: A Next-Generation Sequencing Study. Molecular Psychiatry, 22, 580-584. https://doi.org/10.1038/mp.2016.117

16. Corominas, J., Klein, M., Zayats, T., Rivero, O., Ziegler, G.C., Pauper, M., Neveling, K., Poelmans, G., Jansch, C., Svirin, E., et al. (2018) Identification of ADHD Risk Genes in Extended Pedigrees by Combining Linkage Analysis and Whole Exome Sequencing. Molecular Psychiatry. https://doi.org/10.1038/s41380-018-0210-6

17. Zayats, T., Jacobsen, K.K., Kleppe, R., Jacob, C.P., Kittel-Schneider, S., Ribasés, M., Ramos-Quiroga, J.A., Richarte, V., Casas, M., Mota, N.R., et al. (2016) Exome Chip Analyses in Adult Attention Deficit Hyperactivity Disorder. Translational Psychiatry, 6, e923. https://doi.org/10.1038/tp.2016.196

18. Banaschewski, T., Becker, K., Scherag, S., Franke, B., Coghill, D. (2010) Molecular Genetics of Attention-Deficit/Hyperactivity Disorder: An Overview. European Child \& Adolescent Psychiatry, 19, 237-257. https://doi.org/10.1007/s00787-010-0090-z

19. El-Hazmi, M.A., al-Swailem, A.R., Warsy, A.S., al-Swailem, A.M., Sulaimani, R. and al-Meshari, A.A. (1995) Consanguinity among the Saudi Arabian Population. Journal of Medical Genetics, 32, 623-626. https://doi.org/10.1136/jmg.32.8.623

20. American Psychiatric Association (2013) Diagnostic and Statistical Manual of Mental Disorders. 5th Edition, American Psychiatric Association, Washington DC, 103-106.

21. Parent Electronic Questionnaire. https://docs.google.com/forms/d/e/1FAIpQLSdPkbplKcRaECgUBMtc6aAUQ10Isg0MgElASet9jKiO7VM7yg/vie wform? $\mathrm{c}=0 \& \mathrm{w}=1$

22. American Academy of Pediatrics (2000) Clinical Practice Guideline: Diagnosis and Evaluation of the Child with Attention-Deficit/Hyperactivity Disorder: Committee on Quality Improvement, Subcommittee on Attention-Deficit/Hyperactivity Disorder. Pediatrics, 105, 1158-1570.

23. Kawasaki, Y., Sagara, M., Shibata, Y., Shirouzu, M., Yokoyama, S. and Akiyama, T. (2007) Identification and Characterization of Asef2, a Guanine-Nucleotide Exchange Factor Specific for Rac1 and Cdc42. Oncogene, 26, 7620-7627. https://doi.org/10.1038/sj.onc.1210574

24. Orrù, G. and Carta, M.G. (2018) Genetic Variants Involved in Bipolar Disorder, a Rough Road Ahead. Clinical Practice and Epidemiology in Mental Health, 14, 37-45. https://doi.org/10.2174/1745017901814010037

25. Kim, S.H., Shin, S.Y., Lee, K.Y., Joo, E.J., Song, J.Y., Ahn, Y.M., Lee, Y.H. and Kim, Y.S. (2012) The Genetic Association of DUSP6 with Bipolar Disorder and Its Effect on ERK Activity. Progress in Neuro-Psychopharmacology \& Biological Psychiatry, 37, 41-49. https://doi.org/10.1016/j.pnpbp.2011.11.014

26. Miglis, M.G. (2017) Sleep and Neurologic Disease. In: Sleep and Neurologic Disease, Academic Press, Cambridge, 145 .

27. Tong, L., Ye, Y. and Yan, Q. (2018) The Moderating Roles of Bedtime Activities and Anxiety/Depression in the Relationship between Attention-Deficit/Hyperactivity Disorder Symptoms and Sleep Problems in Children. BMC Psychiatry, 18, 298. https://doi.org/10.1186/s12888-018-1879-4

28. O’Brien, L.M., Holbrook, C.R., Mervis, C.B., Klaus, C.J., Bruner, J.L., Raffield, T.J., Rutherford, J., Mehl, R.C., Wang, M., Tuell, A., et al. (2003) Sleep and Neurobehavioral Characteristics of 5- to 7-Year-Old Children with Parentally Reported Symptoms of Attention-Deficit/Hyperactivity Disorder. Pediatrics, 111, 554-563. 
https://doi.org/10.1542/peds.111.3.554

29. Marcus, M.W., Raji, O.Y., Duffy, S.W., Young, R.P., Hopkins, R.J. and Field, J.K. (2016) Incorporating Epistasis Interaction of Genetic Susceptibility Single Nucleotide Polymorphisms in a Lung Cancer Risk Prediction Model. International Journal of Oncology, 49, 361-370. https://doi.org/10.3892/ijo.2016.3499

30. Tiger, C.F., Fougerousse, F., Grundstrom, G., Velling, T. and Gullberg, D. (2001) Alpha11beta1 Integrin Is a Receptor for Interstitial Collagens Involved in Cell Migration and Collagen Reorganization on Mesenchymal Nonmuscle Cells. Developmental Biology, 237, 116-129. https://doi.org/10.1006/dbio.2001.0363

31. Lesch, K.P., Timmesfeld, N., Renner, T.J., Halperin, R., Röser, C., Nguyen, T.T., Craig, D.W., Romanos, J., Heine, M., Meyer, J., et al. (2008) Molecular Genetics of Adult ADHD: Converging Evidence from Genome-Wide Association and Extended Pedigree Linkage Studies. Journal of Neural Transmission, 115, 1573-1585. https://doi.org/10.1007/s00702-008-0119-3

32. O’Brien, K.M., Orlow, I., Antonescu, C.R., Ballman, K., McCall, L., Dematteo, R. and Engel, L.S. (2013) Gastrointestinal Stromal Tumors: A Case-Only Analysis of Single Nucleotide Polymorphisms and Somatic Mutations. Clinical Sarcoma Research, 3, 12. https://doi.org/10.1186/2045-3329-3-12

33. Lenci, R.E., Rachakonda, P.S., Kubarenko, A.V., Weber, A.N., Brandt, A., Gast, A., Sucker, A., Hemminki, K., Schadendorf, D. and Kumar, R. (2012) Integrin Genes and Susceptibility to Human Melanoma. Mutagenesis, 27, 367-373. https://doi.org/10.1093/mutage/ger090

34. Suzuki, A., Lu, J., Kusakai, G., Kishimoto, A., Ogura, T. and Esumi, H. (2004) ARK5 Is a Tumor Invasion-Associated Factor Downstream of Akt Signaling. Molecular and Cellular Biology, 24, 3526-3535. https://doi.org/10.1128/MCB.24.8.3526-3535.2004

35. Alemany, S., Ribases, M., Vilor-Tejedor, N., Bustamante, M., Sanchez-Mora, C., Bosch R,Richarte, V., Cormand, B., Casas, M., Ramos-Quiroga, J.A., Sunyer, J. (2015) New Suggestive Genetic Loci and Biological Pathways for Attention Function in Adult Attention-Deficit/Hyperactivity Disorder. American Journal of Medical Genetics Part A, 168, 459-470. https://doi.org/10.1002/ajmg.b.32341

36. Ramanan, V.K., Risacher, S.L., Nho, K., Kim, S., Swaminathan, S., Shen, L., Foroud, T.M., Hakonarson, H., Huentelman, M.J., Aisen, P.S., et al. (2014) APOE and BCHE as Modulators of Cerebral Amyloid Deposition: A Florbetapir PET Genome-Wide Association Study. Molecular Psychiatry, 19, 351-357.

https://doi.org/10.1038/mp.2013.19

37. Marszalek, J.R., Weiner, J.A., Farlow, S.J., Chun, J. and Goldstein, L.S. (1999) Novel Dendritic Kinesin Sorting Identified by Different Process Targeting of Two Related Kinesins: KIF21A and KIF21B. The Journal of Cell Biology, 145, 469-479. https://doi.org/10.1083/jcb.145.3.469

38. Hirokawa, N., Niwa, S. and Tanaka, Y. (2010) Molecular Motors in Neurons: Transport Mechanisms and Roles in Brain Function, Development, and Disease. Neuron, 68, 610-638.

https://doi.org/10.1016/j.neuron.2010.09.039

39. Loukola, A., Wedenoja, J., Keskitalo-Vuokko, K., Broms, U., Korhonen, T., Ripatti, S., et al. (2014) Genome-Wide Association Study on Detailed Profiles of Smoking Behavior and Nicotine Dependence in a Twin Sample. Molecular Psychiatry, 19, 615-624. https://doi.org/10.1038/mp.2013.72

40. Li, A., Tian, X., Sung, S.W. and Somlo, S. (2003) Identification of Two Novel Polycystic Kidney Disease-1-Like Genes in Human and Mouse Genomes. Genomics, 81, 596-608. https://doi.org/10.1016/S0888-7543(03)00048-X

41. Yuasa, T., Takakura, A., Denker, B.M., Venugopal, B. and Zhou, J. (2004) Polycystin-1L2 Is a Novel G-Protein-Binding Protein. Genomics, 84, 126-138. https://doi.org/10.1016/j.ygeno.2004.02.008

42. Loebrich, S., Rathje, M., Hager, E., Ataman, B., Harmin, D.A., Greenberg, M.E., et al. (2016) Genomic Mapping and Cellular Expression of Human CPG2 Transcripts in the SYNE1 Gene. Molecular and Cellular Neuros- 
ciences, 71, 46-55. https://doi.org/10.1016/j.mcn.2015.12.007

43. Zhang, X., Xu, R., Zhu, B., Yang, X., Ding, X., Duan, S., Xu, T., Zhuang, Y. and Han, M. (2007) Syne-1 and Syne-2 Play Crucial Roles in Myonuclear Anchorage and Motor Neuron Innervation. Development, 134, 901-908. https://doi.org/10.1242/dev.02783

44. Yu, T.W., Chahrour, M.H. and Coulter, M.E. (2013) Sequencing to Identify Inherited Causes of Autism. Neuron, 77, 259-273. https://doi.org/10.1016/j.neuron.2012.11.002

45. Cross-Disorder Group of the Psychiatric Genomics C. (2013) Identification of Risk Loci with Shared Effects on Five Major Psychiatric Disorders: A Genome-Wide Analysis. The Lancet, 381, 1371-1379. https://doi.org/10.1016/S0140-6736(12)62129-1

46. O’Hurley, G., Busch, C., Fagerberg, L., Hallström, B.M., Stadler, C., Tolf, A., Lundberg, E., Schwenk, J.M., Jirström, K., Bjartell, A., et al. (2015) Analysis of Te Human Prostate-Specific Proteome Defined by Transcriptomics and Antibody-Based Profiling Identifies TMEM79 and ACOXL as Two Putative, Diagnostic Markers in Prostate Cancer. PLoS ONE, 10, e0133449. https://doi.org/10.1371/journal.pone.0133449

47. Shi, S.H., Jan, L.Y. and Jan, Y.N. (2003) Hippocampal Neuronal Polarity Specified by Spatially Localized mpar3/mPar6 and PI 3-Kinase Activity. Cell, 112, 63-75. https://doi.org/10.1016/S0092-8674(02)01249-7

48. Drgon, T., Montoya, I., Johnson, C., Liu, Q.R., Walther, D., Hamer, D., et al. (2009) Genome-Wide Association for Nicotine Dependence and Smoking Cessation Success in NIH Research Volunteers. Molecular Medicine, 15, 21-27.

49. Kim, S.K., Lee, J.Y., Park, H.J., Kim, J.W. and Chung, J.H. (2012) Association Study between Polymorphisms of the PARD3 Gene and Schizophrenia. Experimental and Therapeutic Medicine, 3, 881-885. https://doi.org/10.3892/etm.2012.496

50. Ehlers, C.L., Gizer, I.R., Phillips, E. and Wilhelmsen, K.C. (2010) EEG Spectral Phenotypes: Heritability and Association with Marijuana and Alcohol Dependence in an American Indian Community Study. Drug and Alcohol Dependence, 106, 101-110. https://doi.org/10.1016/j.drugalcdep.2009.07.024

51. Kwan, K.Y., Allchorne, A.J., Vollrath, M.A., Christensen, A.P., Zhang, D.S., Woolf, C.J., et al. (2006) TRPA1 Contributes to Cold, Mechanical, and Chemical Nociception But Is Not Essential for Hair-Cell Transduction. Neuron, 50, 277-289. https://doi.org/10.1016/j.neuron.2006.03.042

52. Nilius, B., Appendino, G. and Owsianik, G. (2012) The Transient Receptor Potential Channel TRPA1: From Gene to Pathophysiology. Pflugers Archiv. European Journal of Physiology, 464, 425-458.

https://doi.org/10.1007/s00424-012-1158-Z

53. Gallo, V., Dijk, F.N., Holloway, J.W., Ring, S.M., Koppelman, G.H., Postma, D.S., et al. (2017) TRPA1 Gene Polymorphisms and Childhood Asthma. Pediatric Allergy and Immunology, 28, 191-198.

https://doi.org/10.1111/pai.12673

54. Muchardt, C., Seeler, J.S., Nirula, A. and Shurland, D.L. (1992) Regulation of Human Immunodeficiency Virus Enhancer Function by PRDII-BF1 and c-rel Gene Products. Journal of Virology, 66, 244-250.

55. Woo, H.J., Yu, C., Kumar, K. and Reifman, J. (2017) Large-Scale Interaction Effects Reveal Missing Heritability in Schizophrenia, Bipolar Disorder and Posttraumatic Stress Disorder. Translational Psychiatry, 7, e1089. https://doi.org/10.1038/tp.2017.61

56. Liu, L., Zhang, L., Li, H.M., Wang, Z.R., Xie, X.F., Mei, J.P., Jin, J.L., Shi, J., Sun, L., Li, S.C., et al. (2017) The SNP-Set Based Association Study Identifies ITGA1 as a Susceptibility Gene of Attention-Deficit/Hyperactivity Disorder in Han Chinese. Translational Psychiatry, 7, e1201. https://doi.org/10.1038/tp.2017.156

57. Douville, P., Seldin, M.F. and Carbonetto, S. (1992) Genetic Mapping of the Integrin Alpha-1 Gene (Vla1) to Mouse Chromosome 13. Genomics, 14, 503-505. https://doi.org/10.1016/S0888-7543(05)80252-6 
58. Bamdad, M., Volle, D., Dastugue, B. and Meiniel, A. (2004) Alphalbeta1-Integrin Is an Essential Signal for Neurite Outgrowth Induced by Thrombospondin Type 1 Repeats of SCO-Spondin. Cell and Tissue Research, 315, 15-25. https://doi.org/10.1007/s00441-003-0793-2

59. Abdulkadir, M., Londono, D., Gordon, D., Fernandez, T.V., Brown, L.W., Cheon, K.A., Coffey, B.J., Elzerman, L., Fremer, C., Fründt, O., Garcia-Delgar, B., et al. (2018) Investigation of Previously Implicated Genetic Variants in Chronic Tic Disorders: A Transmission Disequilibrium Test Approach. European Archives of Psychiatry and Clinical Neuroscience, 268, 301-316. https://doi.org/10.1007/s00406-017-0808-8

60. Kuijpers, T.W., van de Vijver, E., Weterman, M.A., de Boer, M., Tool, A.T., van den Berg, T.K., Moser, M., Jakobs, M.E., Seeger, K., Sanal, O., et al. (2009) LAD-1/Variant Syndrome Is Caused by Mutations in FERMT3. Blood, 113, 4740-4746. https://doi.org/10.1182/blood-2008-10-182154

61. Prasad, A., Merico, D., Thiruvahindrapuram, B., Wei, J., Lionel, A.C., Sato, D., Rickaby, J., Lu, C., Szatmari, P., Roberts, W., et al. (2012) A Discovery Resource of Rare Copy Number Variations in Individuals with Autism Spectrum Disorder. G3, 2, 1665-1685. https://doi.org/10.1534/g3.112.004689

62. SAGE, South Asian Genomes \& Exomes. CSIR Institute of Genomics and Integrated Biology. http://clingen.igib.res.in/sage/ 